\title{
Abundancia y distribución del género Pinus en Capulálpam de Méndez, Sierra Juárez, Oaxaca
}

\section{Abundance and distribution of the genus Pinus in Capulálpam de Méndez, Sierra Juárez, Oaxaca}

\author{
Arianne Ríos-Altamirano', Cecilia Alfonso-Corrado', Víctor Aguirre-Hidalgo', Gregorio Ángeles-Pérez², \\ Magdalena M. Mendoza-Díaz', Viviana Rodríguez-Rivera', Eusebio Roldán-Felix³ y Ricardo Clark-Tapial*
}

1 Universidad de la Sierra Juárez. Instituto de Estudios

Ambientales. Ixtlán de Juárez, Oaxaca, México.

*Autor de correspondencia. rclark@unsij.edu.mx 2 Colegio de Postgraduados. Campus Montecillo. Tex-
coco, Estado de México, México.
3 Unión de Comunidades Productoras Forestales Zapotecas-Chinantecas de la Sierra Juárez. Capulálpam de Méndez, Oaxaca, México.

\section{RESUMEN}

Se analizan los factores ambientales que determinan la abundancia y distribución de las diferentes especies comerciales y aprovechadas del género Pinus en el municipio de Capulálpam de Méndez, Oaxaca. Se utilizó la información de 636 sitios en áreas de aprovechamiento del inventario forestal del municipio llevado a cabo en 2013 para el Plan de Manejo 2015-2022, y 354 puntos adicionales de áreas con uso diferente al forestal. Se obtuvo información sobre la distribución espacial y análisis del nicho ecológico de los pinos, para lo cual se emplearon nueve variables climáticas y cuatro geográficas. Se encontraron 10 especies, de las cuales $P$. patula presentó mayor abundancia y P. chiapensis fue la menor. El análisis de correspondencia canónica indicó que la mayoría de los pinos analizados se asocian en mayor medida con las variables climáticas que con las geográficas a excepción de la latitud. El análisis de nicho mostró que las especies del género Pinus muestran alta marginalidad y baja tolerancia a procesos de cambio ambiental, donde una modificación al hábitat podría restringir o disminuir su abundancia y distribución, siendo $P$. chiapensis la especie de menor tolerancia y $P$. patula la de mayor amplitud de nicho.

PALABRAS ClAVE: amplitud, hábitat, índice de marginalidad, nicho ecológico, tolerancia.

\section{ABSTRACT}

In this study, environmental factors that determine the abundance and distribution of commercial pine species in the municipality of Capulálpam de Mendez, Oaxaca, were analyzed. We used information pertaining to tree species abundance in 636 sites established in harvesting areas from the municipal forest inventory conducted in 2013 for the Management Plan 2015-2022, as well as 354 additional sites located in areas with different land use. Data of the spatial distribution and ecological niche analysis of the species were obtained, for which nine climatic and four geographic variables were used. Ten species of pine were recorded, of which Pinus patula showed the highest abundance and P. chiapensis the lowest. Canonical Correspondence Analysis indicated that most of the pine species are more highly associated with the climatic variables than with geographic variables, except for that of latitude. Niche analysis showed that species of the genus Pinus are highly marginal and of low tolerance to processes of environmental change. Minimal alterations to their habitat could restrict or reduce their abundance and distribution, with $P$. chiapensis being the least tolerant species and $P$. patula the species that presented the broadest niche.

KEYWORDS: amplitude, habitat, outlying mean index, ecological niche, tolerance.

\section{INTRODUCCIÓN}

La abundancia y distribución espacial de los seres vivos en la tierra generalmente no es aleatoria ni estable sino que se rige por la amplitud ecológica o capacidad de tolerancia que presenta cada especie a factores ambientales (Chapman, 1976; Tsiftsis, Tsiripidisa, Karagiannakidoua y Alifragisb, 2008; Thuiller, Gassó, Pino y Vilá, 2012). En el caso de las plantas 
se ha encontrado que su abundancia y distribución se asocia principalmente a factores climáticos, edáficos y geográficos (Kimmins, 2003), así como bióticos, entre los que destaca la interacción competitiva con otras especies y la transformación y pérdida del hábitat que realiza el ser humano debido al cambio de uso de suelo y a las actividades productivas (Meier, Lischke, Schmatz y Zimmermann, 2011) que también generan cambios significativos en las condiciones bióticas y abióticas del ecosistema (Murcia, 1995; Lavergne, Molina y Debussche, 2005).

En ecosistemas templados de México, la abundancia y distribución del género Pinus está influenciada por el clima, el tipo de suelo y la historia biogeográfica de la región (Perry, 1991; Challenger, 1998; Sánchez-González, 2008). Este género es el grupo más importante en el área forestal debido a su historia de aprovechamiento y manejo, sus poblaciones y comunidades ecológicas han variado en una escala espacial y temporal (Richardson et al., 2007). En este contexto, un ecosistema forestal puede ver modificada la cantidad y distribución de especies con potencial productivo debido a la práctica silvícola de seleccionar especies con buenas características morfológicas y de alto valor comercial, por ejemplo, de P. patula a nivel nacional (Castellanos-Bolaños et al., 2008; Ramírez-Santiago, 2015) y de P. halepensis, a nivel internacional (Richardson et al., 2007).

Recientemente, se ha señalado que la abundancia y distribución de los organismos puede cambiar drásticamente en el futuro (Gómez-Mendoza y Arriaga, 2007; van Zonneveld, Koskela, Vinveti y Jarvis, 2009). En México, Gómez-Mendoza y Arriaga (2007) analizaron la sensibilidad de diversas especies de pino al cambio climático bajo dos escenarios, uno severo $\left(22.7^{\circ} \mathrm{C}\right.$ y $\left.660 \mathrm{~mm}\right)$ y otro conservador $\left(21.8^{\circ} \mathrm{C}\right.$ y $721 \mathrm{~mm}$ ) para el año 2050 , concluyen con drásticas reducciones en el área de distribución actual de este taxa. A una escala regional, Cruz-Cárdenas et al. (2016) mencionan que el cambio climático afecta a especies sensibles como P. leiophylla, P. teocote y P. hartwegii y su área de distribución puede disminuir en más de $70 \%$.

En este contexto, comprender la amplitud ecológica y tolerancia de un organismo a diversos factores ambienta- les a lo largo de un gradiente, es un aspecto esencial en ecología aplicada, ya que no solo permite describir su nicho ecológico (Dolédec, Chessel y Gimaret-Carpentier, 2000), sino conocer su grado de especificidad, es decir si son generalistas o especialistas, de acuerdo con los intervalos de tolerancia y distribución de los organismos (Hulbert y White, 2007; Thuiller et al., 2012). El uso del análisis multivariado de marginalidad o outlying mean index (OMI) (Dolédec et al., 2000), permite determinar la amplitud, marginalidad y tolerancia del nicho ecológico de las especies (Tsiftsis et al., 2008; Thuiller et al., 2012), lo cual constituye una herramienta importante para predecir potenciales efectos del cambio climático sobre una especie y planificar labores de reforestación en áreas de distribución potencial de estas.

Este estudio se realizó a una escala de paisaje utilizando el análisis de marginalidad y diversas variables ambientales que afectan la abundancia y distribución de pinos en el municipio de Capulálpam de Méndez, Oaxaca, México, comunidad certificada en el manejo forestal de su bosque. Se conocen las especies presentes en su territorio pero se desconocen los factores ambientales que inciden en su abundancia y distribución.

\section{OBJETIVOS}

El objetivo del trabajo fue determinar la abundancia y distribución de las especies de pinos y evaluar la sensibilidad de su nicho ecológico con la finalidad de realizar recomendaciones para la conservación y manejo forestal en el municipio de Capulálpam de Méndez.

\section{MATERIALES Y MÉTODOS}

\section{Descripción del área de estudio}

El área de estudio se limitó al municipio de Capulálpam de Méndez, ubicado en la Sierra Juárez al Norte del estado de Oaxaca. Se localiza entre las coordenadas $96^{\circ} 27^{\prime}$ de longitud oeste y $17^{\circ} 18^{\prime}$ latitud Norte. Cuenta con una superficie de 3849 ha (Fig. 1), de las cuales 2557.51 ha se encuentran bajo aprovechamiento forestal (Unión de Comunidades Productoras Forestales Zapotecos-Chinan- 


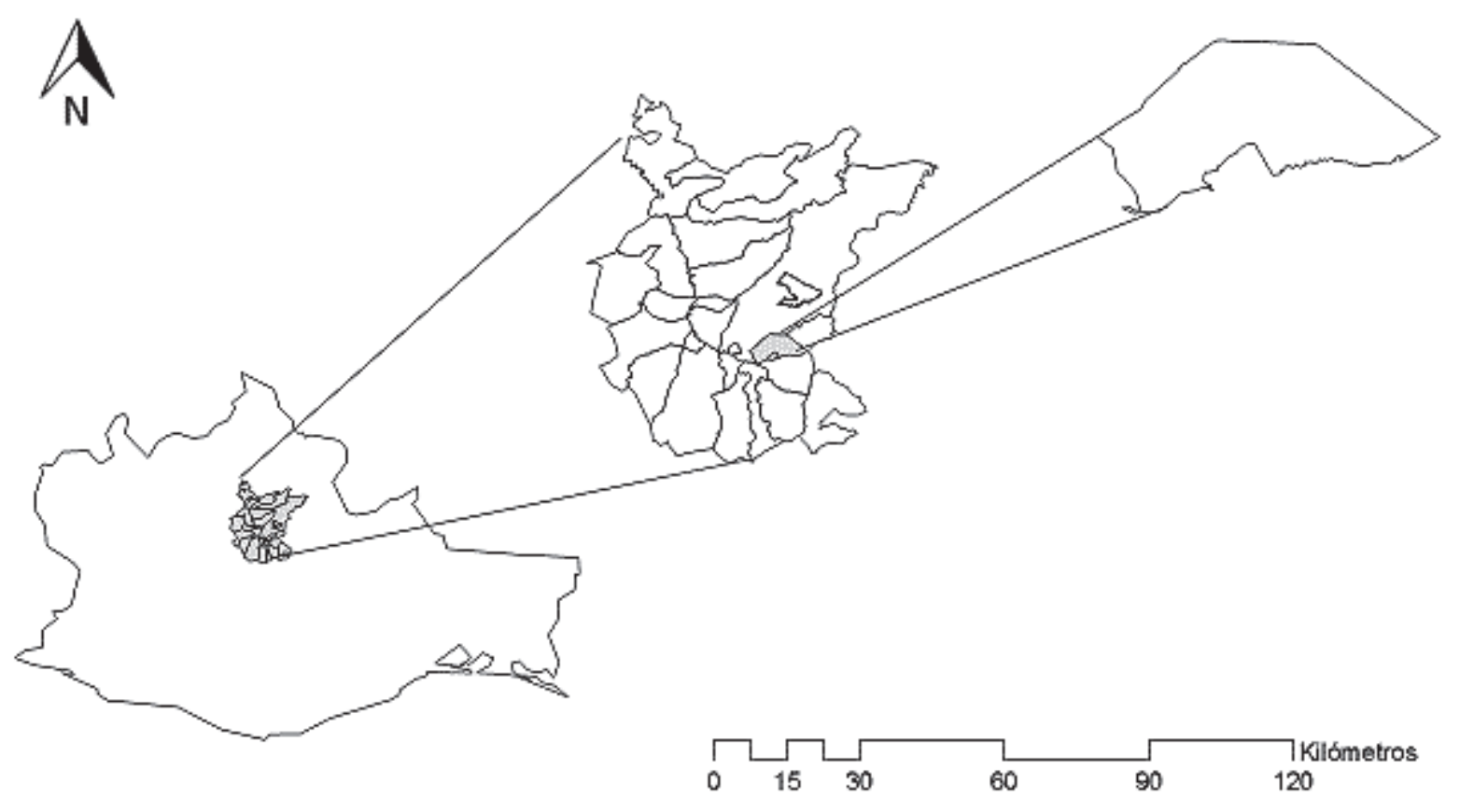

Figura 1. Ubicación del municipio de Capulálpam de Méndez, en el Distrito de Ixtlán, Oaxaca, México.

tecos de la Sierra Juárez [Uzachi], 2003). Limita al norte con los terrenos comunales de Ixtlán de Juárez y San Juan Tepanzacoalcos, al sur con terrenos comunales de San Juan Juquila Vijanos, Santiago Xiacui y pueblos mancomunados (Lachatao, Amatlán y Yavesía); al este con la comunidad de San Miguel Yotao y al oeste con terrenos comunales de Santa María Yahuiche (Uzachi, 2003).

Los tipos de suelo que predominan son Cambisol, Litosol y Redzina (Plan de Desarrollo Municipal [PDM], 2009). El clima, según la clasificación de Köppen modificada por E. García, es $\mathrm{C}\left(\mathrm{w}_{2}\right)$ (w) big, definido como un clima templado subhúmedo, el más húmedo de los subhúmedos con lluvias abundantes en verano. La precipitación total es de $1115 \mathrm{~mm}$ anuales y los meses con mayor intensidad van de junio hasta octubre, mientras que la temperatura promedio anual es de $15.2^{\circ} \mathrm{C}$ (PDM, 2009). La altitud varía de los $2000 \mathrm{~m}$ a los $3000 \mathrm{~m}$ snm, con una altitud promedio de $2120 \mathrm{~m}$ snm (PDM, 2009). Los tipos de vegetación que se encuentran son bosque de pino $(P$. leiophylla-P. lawsonii), pino-encino (Pinus lawsoniiQuercus urbanii-Quercus crassifolia), latifoliadas, asociaciones de bosques de encino con matorrales secundarios
(Quercus-Acacia), y en algunas partes se encuentran pinos con elementos de bosque mesófilo de montaña donde ocurren los géneros Quercus, Oreopanax, Pinus y Dendropanax (Uzachi, 2003; PDM, 2009).

\section{Manejo forestal de la comunidad}

En la década de 1950 el bosque de Capulálpam de Méndez fue concesionado 25 años, por decreto presidencial, a la empresa Fábricas de Papel Tuxtepec (Fapatux) para el aprovechamiento de madera del género Pinus, quien utilizó el Método Mexicano de Ordenación de Montes (MMOM) (Uzachi, 2003) también conocido por Método Mexicano de Ordenación de Bosques Irregulares (MMOBI) (Hernández-Díaz, Corral-Rivas, Quiñones-Chávez, Bacon-Sobbe y Vargas-Larreta, 2008). A principios de la década de 1980 la concesión llegó a su fin y las comunidades indígenas recuperaron el control de sus bosques, y a partir de entonces desarrollaron un manejo forestal comunitario (Bray y Merino, 2004). Este tipo de manejo forestal consistió en el Método Desarrollo Silvícola (MDS) con la aplicación de cortas de regeneración de árboles padre, en combinación con 
el MMOBI, utilizando como método de regeneración el de selección grupal. A partir del año 2003 el método de regeneración de árboles padre fue sustituido por el sistema de corta de regeneración de matarrasa en franjas (Uzachi, 2003).

\section{Obtención de datos de abundancia}

Se utilizó información de abundancia proporcionada por la Unión de Comunidades Productoras Forestales Zapotecos-Chinantecos de la Sierra Juárez (Uzachi). Esta consistió en una base de datos con información de abundancia de especies de 636 sitios de muestreo ubicados en áreas de aprovechamiento forestal, obtenidos de censos en parcelas circulares de $1000 \mathrm{~m}^{2}$, que forman parte del inventario forestal del Plan de Manejo Forestal 2015-2022. Adicionalmente, se incorporaron 354 sitios de muestreo de áreas con uso diferente al forestal (agrícola, urbano, turístico), con la finalidad de tener representado todo el municipio. En total se trabajó con una base de datos con 990 sitios con datos de abundancia total y promedio por especie; así como cuatro variables del sitio: coordenadas geográficas (latitud, X y longitud, Y), altitud (ALT) y pendiente (\%P). Para analizar las diferencias en la abundancia entre parcelas se realizó un análisis Anova Kruskall-Wallis en rangos y prueba múltiple de contraste de Tukey usando el programa XLSTAT ${ }^{\mathrm{TM}}$ (Addinsof, 2014).

\section{Distribución espacial}

El análisis espacial fue realizado usando los datos de coordenadas geográficas de cada parcela en el programa PAST $^{\text {TM }} 2.17$ (Hammer, Harper y Ryan, 2001), para analizar la distribución espacial de Ripley's $\mathrm{K}$ de cada especie. Cuando la función empírica alcanza valores superiores al modelo nulo a una determinada distancia, implica la existencia de un patrón espacial en agregados significativos a esa distancia; por el contrario, cuando aparece por abajo, implica la existencia de un patrón espacial regular. Adicionalmente, se realizó una prueba de uniformidad de Rayleigh con la que se estimó la orientación cardinal preferente de cada especie, usando el programa PAST ${ }^{\mathrm{TM}} 2.17$ (Hammer et al., 2001).

\section{Obtención del modelo digital de elevación}

La ubicación específica de cada especie en Capulálpam de Méndez se situó en un modelo digital de elevación (MDE) del municipio, generado a partir de las curvas de nivel (equidistancia $40 \mathrm{~m}$ ) del conjunto de datos vectoriales de las cartas topográficas E14D38, escala 1:50 000 (Instituto Nacional de Estadística, Geografía e Informática [Inegi], 2001). Este procedimiento se desarrolló mediante el sistema de información geográfica ArcGis 9.3 ${ }^{\mathrm{TM}}$ (Esri, 2000) en la extensión 3D Analyst Tools. El MDE se realizó con el sistema de coordenadas UTM, zona $14 \mathrm{~N}$, con datum de referencia WGS84 y resolución de $15 \mathrm{~m}$.

\section{Análisis de datos del nicho ecológico}

Para realizar el análisis de marginalidad (Outlying Mean Index, OMI) se empleó el software ADE-4 ${ }^{\mathrm{TM}}$ (Thioulouse, Chessel, Dolédec y Olivier, 1997), donde se utilizaron datos de las variables del sitio y nueve variables climáticas obtenidas de la base de datos WorldClim de la página electrónica http://www.worldclim.org_(Hijmans et al., 2005), debido a que el municipio carece de datos climáticos: precipitación máxima (Pmax), precipitación máxima del cuartil más caluroso (Pmts), precipitación mínima (Pmin), precipitación máxima del cuartil más lluvioso (Pmlv), temperatura promedio anual (Tpm), temperatura máxima del cuartil más frío (Tmf), temperatura máxima promedio (Tmaxp), temperatura mínima promedio (Tminp) y desviación estándar de la temperatura (Tdv) y c) los datos de la abundancia de cada especie.

El análisis utiliza dos matrices: una botánica que contiene la abundancia de la especie (en renglones) y los sitios (en columnas); y otra matriz ambiental (climática más geográfica) con valores de las variables ambientales (en renglones) y mismos $n$ sitios (en columnas). Como primer paso, la matriz ambiental fue analizada en sus componentes principales (ACP) con la finalidad de ordenar los sitios en función de las variables ambientales. El OMI organiza la inercia asociada con la distribución de una especie en tres términos: 1) el OMI, que estima la marginalidad de una determinada especie, es decir, la distancia entre las condiciones encontradas en la zona donde se 
encuentra y las condiciones medias del hábitat en toda el área de estudio. Valores altos de OMI indican que las especies tienen nichos marginales, lo que corresponde a especies que ocurren en hábitats atípicos; 2) la amplitud del nicho o tolerancia de la especie corresponde a la dispersión de los sitios de muestreo que contienen una especie a lo largo de un gradiente ambiental. Valores bajos de tolerancia significa que la especie cuenta con un intervalo limitado de condiciones (e.g. especies especialistas), mientras que los valores altos implican que una especie se distribuye a través de condiciones ambientales muy variables (e.g. especies generalistas); finalmente, 3 la tolerancia residual, que representa la variación en el hábitat no explicada por las variables explicativas del estudio. Valores bajos de este parámetro, indican que las variables analizadas explican la distribución de una especie, mientras que valores altos indican una débil relación (Dolédec et al., 2000; Tsiftsis et al., 2008).

Se utilizó una prueba de Monte Carlo para comparar la marginalidad observada de la especie versus su distribución a través de 10000 permutaciones al azar y una significancia de $P=0.05$, bajo la hipótesis nula de que el ambiente no afecta la abundancia de la especie.

\section{Resultados}

\section{Abundancia de las especies}

Se encontró un total de 10 especies de pinos, de las cuales una tiene dos variedades, P. pseudostrobus (Tabla 1). Pinus patula presentó la mayor abundancia (4814 individuos) y presencia en los puntos de muestreo $(58 \%)$, seguida en abundancia de P. pseudostrobus var. pseudostrobus (1253 individuos) y $P$. douglasiana (1385 individuos), mientras que $P$. chiapensis y $P$. leiophylla fueron las especies con menor abundancia y ocurrencia (Tabla 1; Fig. 2).

En general, las parcelas presentaron una baja diversidad de coníferas. El número promedio de especies presentes por sitio fue de $1.38 \pm 0.85$. El mayor porcentaje de las parcelas registró, de manera predominante, la presencia de una, dos o tres especies $(48 \%, 30 \%$ y $20.94 \%$, respectivamente); lo que representó $98.9 \%$ del total analizado.
De manera poco frecuente, se ubicaron parcelas con presencia de cuatro a cinco especies $(0.91 \%$ y $0.15 \%$ respectivamente). Este resultado se relaciona con la baja abundancia total y promedio de pinos presentes en las parcelas (Tabla 1) en donde predominaron sitios de muestreo con ausencia de pinos (49\% de los sitios analizados) y sitios con menos de 10 individuos en $1000 \mathrm{~m}^{2}$ (27\%), siendo escasas las parcelas con más de 50 individuos $(<$ $3 \%$ de los sitios analizados).

\section{Distribución de las especies de pino en Capulálpam de Méndez}

De manera general, la mayoría de las especies mostró una distribución espacial significativa $(\mathrm{R}=1.45 ; \mathrm{P}<0.016)$ dispersa a cortas distancias $(<$ de $300 \mathrm{~m}$ ) y agregada a grandes distancias (de $300 \mathrm{~m}$ en adelante), con excepción de $P$. leiophylla y $P$. rudis que mostraron una distribución espacial al azar $(\mathrm{R}=1.01 ; \mathrm{P}<0.007)$.

Utilizando como base el modelo de elevación digital, se definieron tres estratos altitudinales para Capulálpam de Méndez: a) la zona baja que comprende de los $1680 \mathrm{~m}$ a $2205 \mathrm{~m} \mathrm{snm}$; b) la zona media de $2205 \mathrm{~m}$ a $2730 \mathrm{~m} \mathrm{snm}$ y c) la zona alta con altitudes de $2730 \mathrm{~m}$ a $3080 \mathrm{~m} \mathrm{snm}$, dentro de los cuales se ubicó la distribución y presencia de cada especie. Para brindar una mejor interpretación de los resultados se describe el patrón de distribución a nivel de cada una de ellas (Fig. 3a-j).

$P$. ayacahuite tuvo una mayor presencia en la zona media del municipio (Fig. 3a), con mayor preferencia por la orientación $\mathrm{NE}\left(81.4^{\circ}, \mathrm{R}=0.78, \mathrm{P}<0.005\right)$. La especie se registró en zonas húmedas localizadas a una altitud que va de los $2205 \mathrm{~m}$ a los $2730 \mathrm{~m}$ snm. No se encontró en la zona baja, mientras que su presencia en la zona alta es mínima. Las especies que mostraron una asociación con $P$. ayacahuite fueron $P$. patula y $P$. pseudostrobus var. pseudostrobus presentes en $65 \%$ y $23 \%$ de los sitios respectivamente y en menor asociación con $P$. douglasiana (9\%), P. leiophylla $(0.7 \%)$ y $P$. rudis $(0.5 \%)$, mientras que en $11 \%$ de los sitios se presentó de manera exclusiva.

$P$. douglasiana mostró un patrón similar a $P$. ayacahuite, al presentarse con mayor frecuencia en la zona 
Tabla 1. Abundancia total (At) y promedio (Ap) de las especies de Pinus en Capulálpam de Méndez.

\begin{tabular}{lccc}
\hline Especie & $\begin{array}{c}\text { At en el } \\
\text { municipio }\end{array}$ & $\begin{array}{c}\text { Ap en } \\
1000 \mathrm{~m}^{2}\end{array}$ & Abreviaturas \\
\hline P. ayacahuite C.Ehrenb. ex Schltdl. & 152 & $0.18 \pm 0.02^{\mathrm{a}}$ & Pah \\
P. douglasiana Martínez & 1385 & $1.42 \pm 0.14^{\mathrm{b}}$ & Pdg \\
P. leiophylla Schiede ex Schltdl. et Cham. & 57 & $0.05 \pm 0.02^{\mathrm{c}}$ & Plp \\
P. pseudostrobus Lindl. var. oaxacana* & 464 & $0.08 \pm 0.01^{\mathrm{c}}$ & Ppso \\
P. patula Schltdl. \& Cham. var. Patula & 4986 & $5.06 \pm 0.32^{\mathrm{d}}$ & Ppl \\
P. pseudostrobus Lindl. var. pseudostrobus & 1717 & $1.85 \pm 0.21^{\mathrm{b}}$ & Pps \\
P. rudis Lindl. & 285 & $0.29 \pm 0.10^{c}$ & Phd \\
P. teocote Schltdl. \& Cham . & 811 & $0.83 \pm 0.08^{\mathrm{e}}$ & Ptc \\
Pinus lawsonii Roezl ex Gordon et Glend & 54 & $0.04 \pm 0.008^{c}$ & Plw \\
Pinus devoniana Lindl. & 46 & $0.03 \pm 0.007^{c}$ & Pd \\
Pinus chiapensis (Martínez) Andresen & 10 & $0.01 \pm 0.009^{c}$ & Pch \\
\hline
\end{tabular}

At-suma de todos los individuos de la especie en los sitios muestreados y Ap-número estimado con base en el promedio por 1000 m².

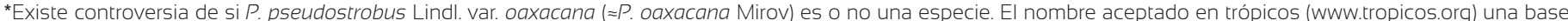
de datos taxonómica internacional, es Pinus pseudostrobus var. apulcensis (Lindl.) Shaw, no obstante, Conafor (2007a) la reconoce con el nombre brindado por Uzachi.

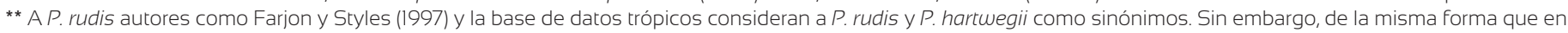

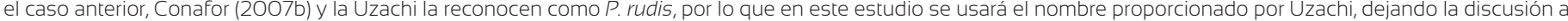
futuros trabajos taxonómicos o moleculares, lo cual no es objetivo de este estudio.

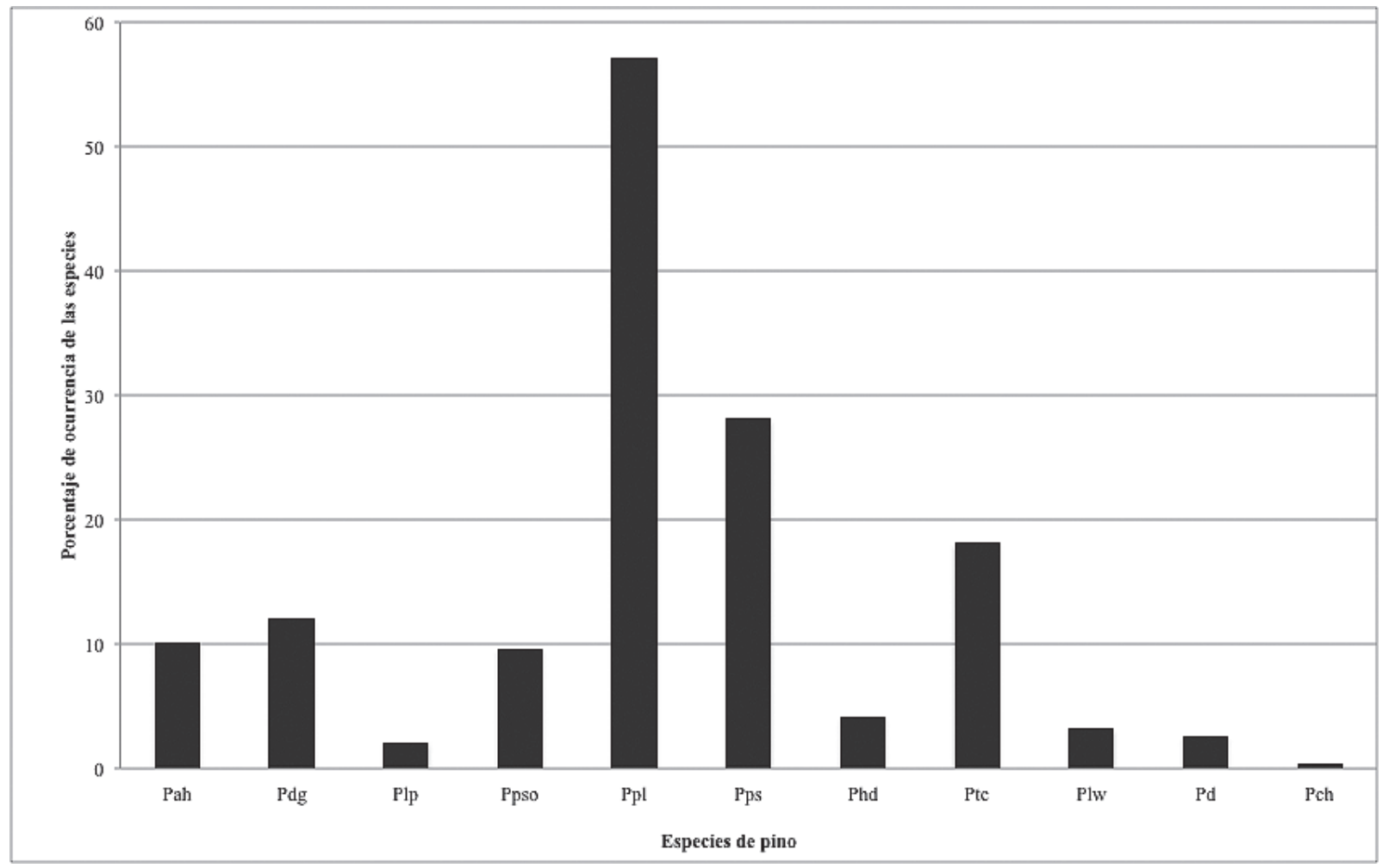

Figura 2. Porcentaje de sitios con ocurrencia de especies de Pinus en Capulálpam de Méndez. 
a)

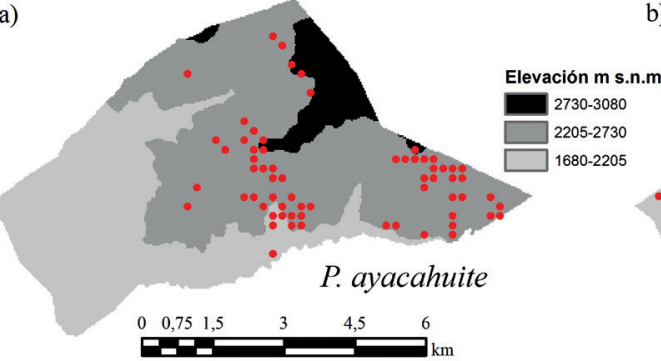

b)

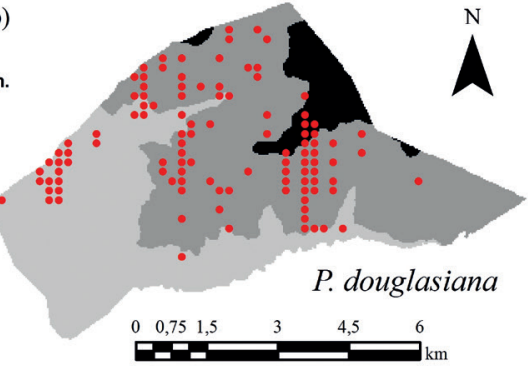

d)
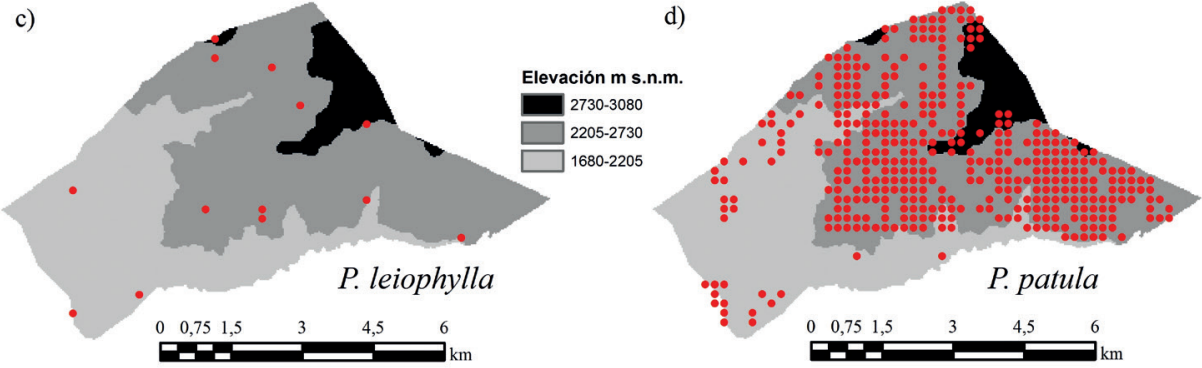

e)

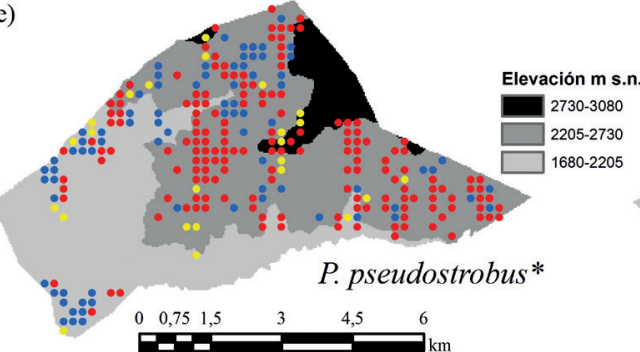

f)

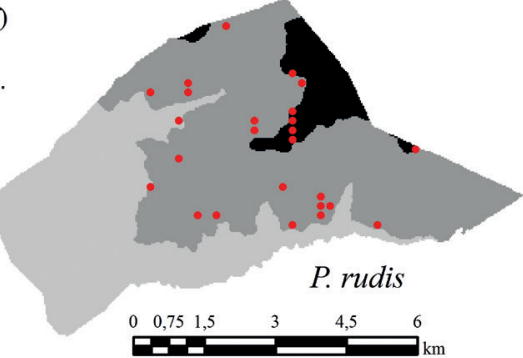

g)

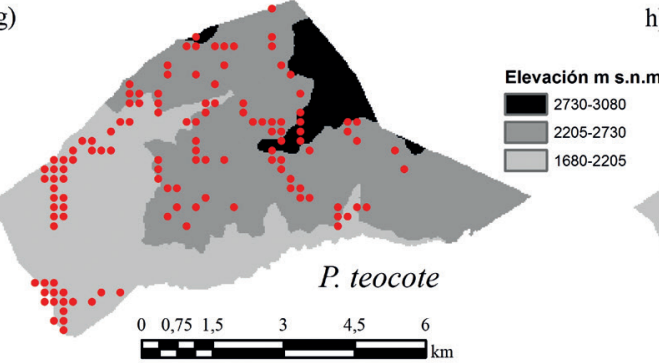

h)
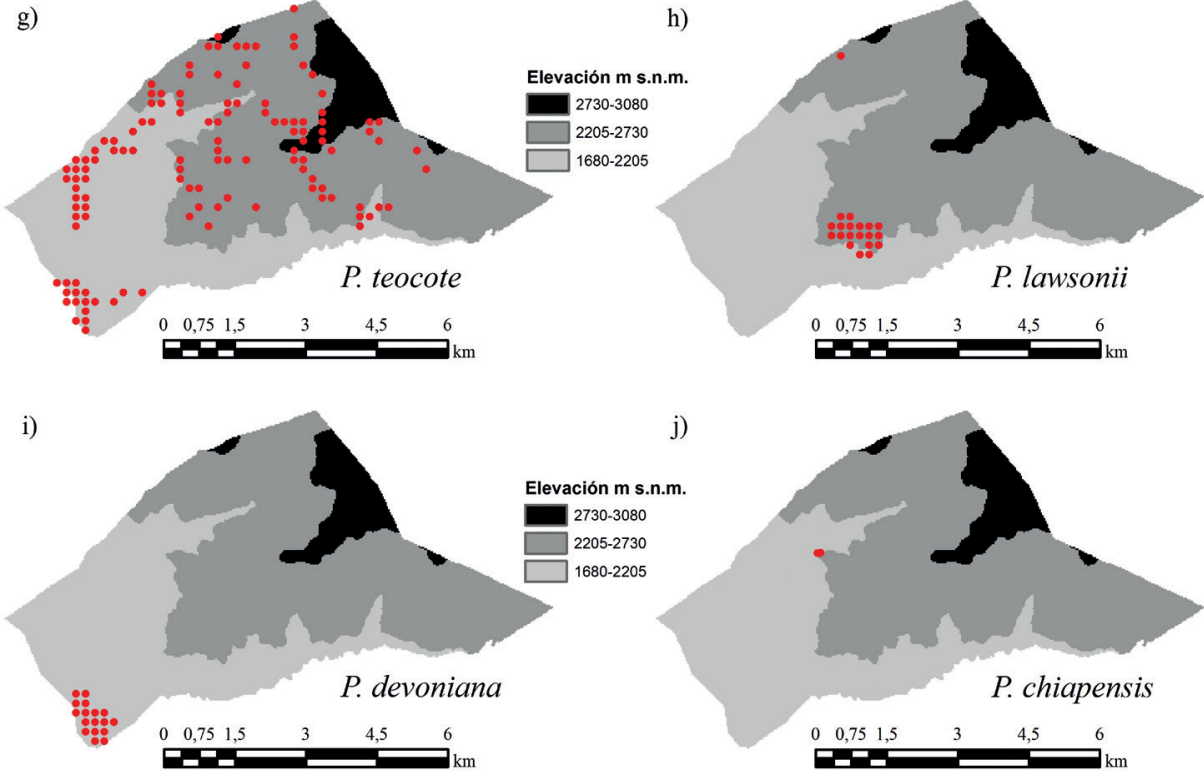

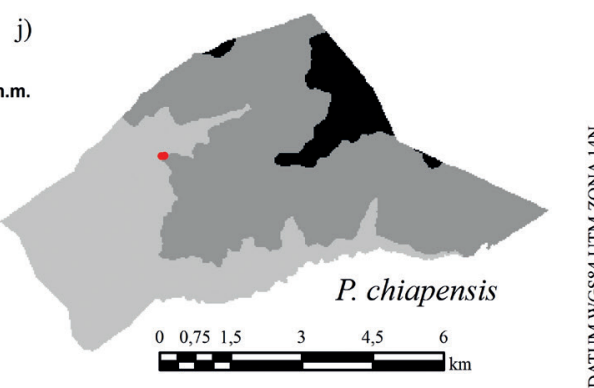

Figura 3. Distribución de las especies de Pinus en Capulálpam de Méndez.

*Se indica a P. pseudostrobus var. pseudostrobus con el símbolo de color rojo, a P. pseudostrobus var. oaxacana de color azul y la presencia de ambas en el mismo sitio se indica de color amarillo. 
media (2205 m - 2730 m snm) (Fig. 3b). La especie mostró preferencia por laderas y la orientación $\mathrm{NE}\left(82.3^{\circ}, \mathrm{R}=\right.$ $0.77, \mathrm{p}<0.002)$. Este pino se encontró solo en $69 \%$ de las parcelas y cuando se encuentra en asociación es con $P$. patula.

P. leiophylla se registró solo en 12 sitios que corresponden a laderas de la zona media del municipio (Fig. 3c). Su distribución espacial fue significativa aleatoria y mostró una orientación preferente por el E $\left(93^{\circ}, \mathrm{R}=0.61, \mathrm{p}<0.009\right)$. Su asociación con otros pinos fue baja, menor a $0.5 \%$ de las parcelas con P. patula, P. ayacahuite, P. teocote, P. pseudostrobus var. pseudostrobus y P. douglasiana.

\section{P. pseudostrobus var. oaxacana (Pinus oaxacana} Mirov) mostró alta presencia y abundancia en la zona baja y media (Fig. 3d) y una orientación NE $\left(78.9^{\circ}, \mathrm{R}=\right.$ $0.72, \mathrm{p}<0.005)$. Este pino se asoció principalmente con $P$. teocote con quién estuvo presente en $45 \%$ de los sitios, con P. pseudostrobus var. pseudostrobus en $37 \%$ de los sitios y con $P$. patula en $30 \%$ de las parcelas. En menor porcentaje se asoció con $P$. douglasiana y $P$. ayacahuite en $3 \%$ y $1.7 \%$ de los sitios, respectivamente. En $33 \%$ de las parcelas muestreadas la especie se presentó de manera exclusiva.

P. patula fue la especie más abundante de todas y su distribución se concentró principalmente en la parte media, en la parte baja se presentó en pequeñas porciones y en la parte alta se le encontró alrededor de los $2900 \mathrm{~m}$ (Fig. 3e), ubicándose en las laderas y sitios con una orientación preferente $\mathrm{NE}\left(38^{\circ}, \mathrm{R}=0.53, \mathrm{p}<0.003\right)$. $P$. patula se encontró asociada preferentemente con $P$. pseudostrobus var. pseudostrobus (42\%) y P. ayacahuite (13\%).

P. pseudostrobus var. pseudostrobus se registró en las tres zonas (Fig. 3d), con excepción de las altitudes máxima (3080 m snm) y mínima (1680 m snm). Esta especie mostró preferencia por sitios con buena exposición, orientados preferentemente al $\mathrm{NE}\left(73^{\circ}, \mathrm{R}=0.77, \mathrm{p}<\right.$ $0.003)$. Se encontró asociada en mayor medida con $P$. patula (64\%), P. teocote (25\%), P. pseudostrobus var. oaxacana $(22 \%)$ y P. ayacabuite $(10 \%)$.

$P$. rudis, al igual que $P$. leiophylla, presentó baja abundancia (Fig. 3f). Esta especie se encontró con mayor frecuencia en altitudes elevadas ya que a pesar de encontrarse desde los $2200 \mathrm{~m}$ snm con unos pocos individuos, su abundancia se incrementó a partir de los 2500 m - 2800 $\mathrm{m}$ snm donde mostró una orientación preferente NE $\left(82^{\circ}\right.$, $\mathrm{R}=0.71, \mathrm{p}<0.001) . P$. rudis se encontró asociada con $P$. teocote (37.5\%), P. patula (29\%), P. pseudostrobus var. pseudostrobus $(17 \%)$, así como con $P$. ayacabuite y $P$. douglasiana en $12.5 \%$ de los sitios.

P. teocote se registró en solo $18 \%$ de los sitios analizados (Fig. 3g); no obstante, es de las especies más abundantes en el municipio, particularmente en la parte baja y media, con presencia menos abundante en la parte alta donde mostró una orientación preferente $\mathrm{NE}\left(66^{\circ}, \mathrm{R}=\right.$ $0.43, \mathrm{p}<0.05)$. Se le encontró asociada con P. pseudostrobus var. pseudostrobus (45\%), P. patula (33\%), y en menor medida con $P$. rudis ( $8 \%$ ). Esta especie estuvo sola en $41 \%$ de los sitios analizados.

P. lawsonii presentó baja abundancia (Fig. 2) y ocurrencia (Fig. 3h) en el municipio. Esta especie se encontró con mayor frecuencia a altitudes medias, ya que a pesar de encontrarse desde los $2100 \mathrm{~m}$ snm con unos pocos individuos, su abundancia máxima se encontró entre los 2250 m y 2400 m snm, donde mostró una orientación preferente $\mathrm{NE}\left(85.5^{\circ}, \mathrm{R}=0.83, \mathrm{p}<0.001\right)$. P. lawsonii se encontró asociada con P. patula (60\%), P. pseudostrobus var. pseudostrobus (35\%), P. teocote (30\%) y en menor ocurrencia con P. douglasiana (10\%).

P. devoniana solo se registró en 16 parcelas al SE del municipio (Fig. 3i), donde predomina en laderas de la parte baja a una altitud promedio de $1989 \mathrm{~m}$ snm, con una distribución espacial significativa aleatoria a cortas y grandes distancias y uniforme a distancias intermedias y una orientación promedio $\mathrm{NE}\left(73^{\circ}, \mathrm{R}=0.88, \mathrm{p}<0.001\right)$. Se asoció principalmente con $P$. pseudostrobus var. pseudostrobus (75\%) y en menor medida con P. patula (19\%) y P. teocote, (35\%).

P. chiapensis mostró presencia solamente en dos sitios del municipio con abundancia muy baja de individuos (Fig. 3j), a una altitud promedio de $2210 \mathrm{~m} \mathrm{snm}$, en un patrón agregado y con orientación SO. P. patula fue la única especie asociada en ambos sitios. 


\section{Análisis de nicho ecológico}

El análisis del índice de marginalidad indicó un efecto directo de las variables ambientales sobre la abundancia y la distribución de las especies de pinos en Capulálpam de Méndez. Ochenta por ciento de las especies son influenciadas significativamente por las variables ambientales analizadas. Las coníferas con mayores valores del OMI fueron $P$. ayacahuite, $P$. chiapensis, $P$. devoniana, $P$. lawsonii y $P$. rudis mientras que las cinco especies restantes mostraron un valor menor a la unidad (Tabla 2). Los valores del índice de tolerancia mostraron que los pinos con mayor tolerancia o amplitud de nicho en el municipio fueron P. teocote, $P$. patula y $P$. rudis, mientras que las menos tolerantes fueron P. chiapensis, P. lawsonii y P. devoniana (Tabla 2). Sesenta y cuatro por ciento de las coníferas presentaron una tolerancia baja (valores menores a la media), lo que indicó un nicho muy sensible a cambios, es decir, un nicho con baja amplitud o muy especializado. Se encontró que las coníferas mostraron un bajo porcentaje de tolerancia residual, lo cual indica que los parámetros de hábitat analizados explicaron satisfactoriamente su distribución (Tabla 2).

Los primeros dos ejes del análisis OMI explicaron 90\% de la varianza obtenida (Fig. 4), en el inciso a de la figura 4 se muestra que los pinos responden de manera diferente a las variables geográficas y climáticas de la figura 4 inciso $b$, mostrando una correlación positiva o negativa significativa. La posición de las especies a lo largo de los dos primeros ejes del OMI permitió separar claramente dos grupos de coníferas; el primero (parte izquierda de la figura 4a) que consistió en pinos asociados a variables geográficas (latitud, longitud y altitud) y las variables climáticas de precipitación, mientras que el segundo grupo (parte derecha de la figura 4a) mostró a especies asociadas con las variables climáticas de temperatura.

\section{DISCUSIÓN}

Las diez especies estudiadas en Capulálpam de Méndez, representan $83 \%$ de los pinos documentados para la Sie-
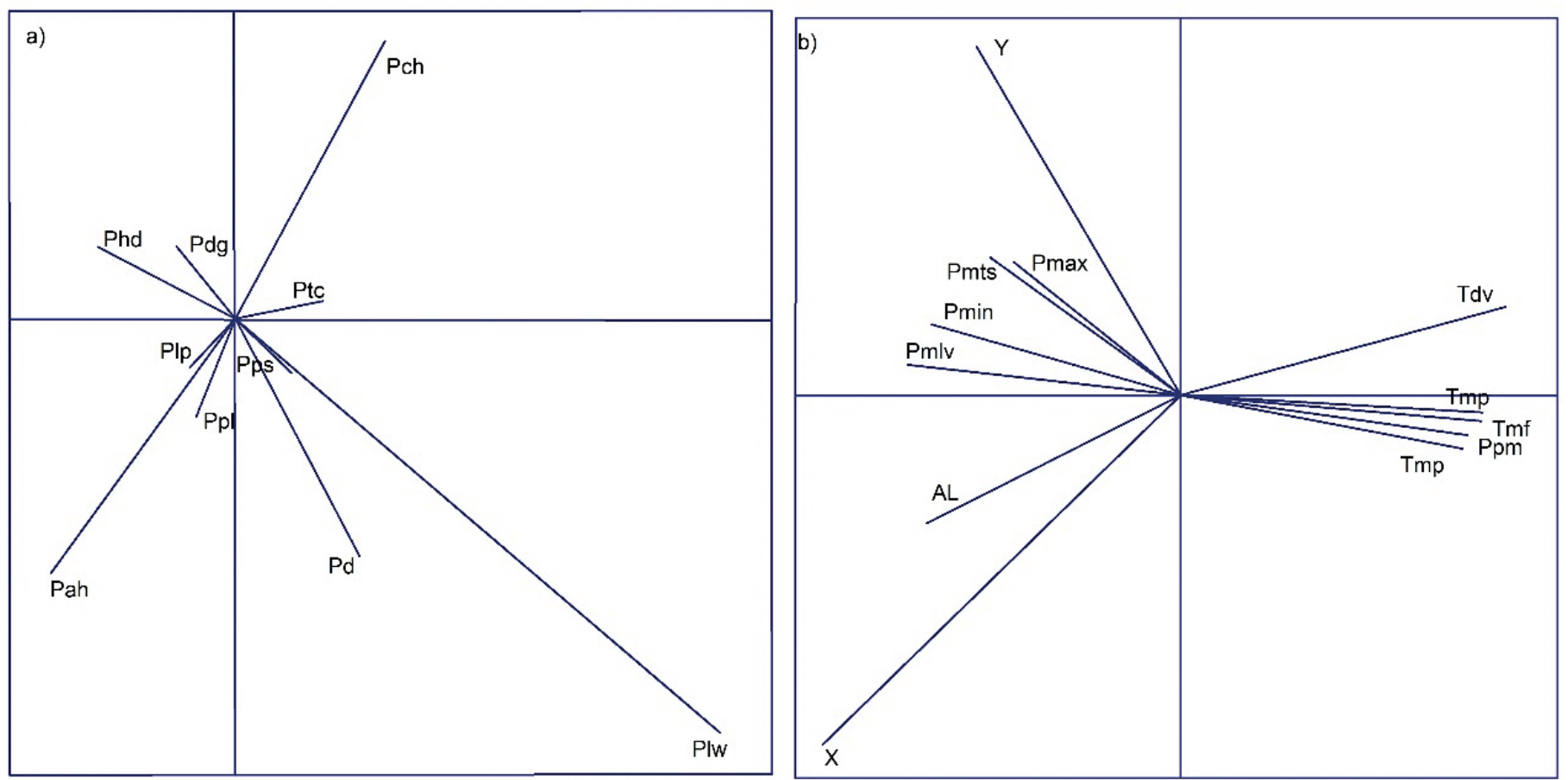

Figura 4. Valores canónicos de las variables y su efecto sobre la distribución de las especies de Pinus en Capulálpam de Méndez.

a) Distribución de las especies en los primeros dos ejes del análisis de marginalidad y b) peso canónico de las variables ambientales. La longitud de las flechas describe la importancia relativa de cada variable analizada y la dirección de la flecha indica la correlación entre variables. Ver tabla 1 para abreviatura de las especies y sección datos de nicho ecológico en métodos para la abreviatura de las variables. 
Tabla 2. Parámetros de nicho para las especies de Pinus en Capulálpam de Méndez.

\begin{tabular}{lccccc}
\hline Especie & Inercia & OMl & T1 & T2 & P \\
\hline Pah & 7.788 & 3.394 & 2.277 & 2.281 & $*$ \\
Pdg & 8.778 & 0.374 & 2.475 & 5.931 & $*$ \\
Plp & 6.576 & 0.222 & 3.545 & 2.813 & NS \\
Ppso & 16.484 & 2.071 & 2.566 & 3.931 & $*$ \\
Ppl & 8.354 & 0.202 & 4.232 & 8.026 & $*$ \\
Pps & 10.414 & 0.313 & 2.079 & 2.085 & $*$ \\
Phd & 6.394 & 1.899 & 1.970 & 8.06 & NS \\
Prc & 11.410 & 0.707 & 2.657 & 8.057 & $*$ \\
Plw & 2.414 & 2.111 & 0.265 & 0.270 & NS \\
Pd & 2.283 & 2.710 & 0.657 & 0.224 & $*$ \\
Pch & 2.808 & 2.808 & 0.000 & 0.000 & NS \\
\hline
\end{tabular}

Nombres de especies y abreviaturas se encuentran en Tabla 1. OMl = Índice promedio de marginalidad; $T T 1$ = Índice de tolerancia; $T 2$ = Porcentaje de tolerancia residual; $P=$ representa el número de simulaciones (1000) que representan un mayor valor del observado en el OMI (NS = no significativo a un valor de $\mathrm{P}>0.05$ ).

rra Norte de Oaxaca y $71 \%$ a nivel estatal (14 especies de acuerdo con Del Castillo, Pérez de la Rosa, Vargas-Amado y Rivera-García, 2004). Este es un porcentaje de especies alto si se compara con regiones de bosques templados de mayor extensión tanto en el estado de Oaxaca (Del Castillo et al., 2004), como a nivel nacional (e.g. MárquezLinares, González-Elizondo y Álvarez-Zagoya, 1999; Enríquez-Enríquez, Koch y González-Elizondo, 2003; Díaz, Sosa-Ramírez y Pérez-Salicrup, 2012). De las especies presentes, $P$. patula mostró la mayor abundancia y distribución en el municipio, tres veces más que $P$. pseudostrobus var. pseudostrobus, que es la segunda especie en abundancia. Este resultado es congruente con su uso al ser $P$. patula y $P$. pseudostrobus las especies más aprovechadas forestalmente en México (Perry, 1991; Bermejo y Pontones, 1999; Castellanos-Bolaños et al., 2008).

En Oaxaca, estas dos especies son sumamente apreciadas en el sector forestal, particularmente desde el punto de vista económico, producto de las buenas características morfológicas de sus fustes (Del Castillo et al., 2004;
Comisión Nacional Forestal [Conafor], 2007a). En Ixtlán de Juárez, predio comunal colindante con Capulálpam de Méndez, se ha encontrado que $P$. patula y $P$. pseudostrobus presentan una mayor abundancia y distribución con respecto a otras especies (Hipólito Vázquez datos no publicados). De igual manera, estas dos especies son registradas por Castellanos-Bolaños et al. (2008) como las de mayor distribución en la Sierra Juárez. Esto puede ser debido a que se impulsa un proceso de selección o reemplazo de especies no comerciales por aquellas con potencial económico en las comunidades forestales de la sierra.

La modificación de la estructura forestal es una consecuencia directa del manejo forestal (Castellanos-Bolaños et al., 2008; Ramírez-Santiago, 2015), lo cual no solo puede favorecer la abundancia y distribución de las coníferas de interés comercial, sino afectar a otras especies (SolísMoreno et al., 2006; Richardson et al., 2007; Jardel, 2012). La alta presencia de $P$. patula en el municipio con fines de manejo forestal es ejemplo de una práctica común en el ámbito forestal que conduce a la modificación de la estructura del bosque (Perry, Graham y Richardson, 1998; Richardson et al., 2007). Por consiguiente, una posible respuesta a la baja abundancia y distribución de las especies de menor valor comercial (e.g. P. teocote, P. leiophylla y P. rudis) en la región, sea producto de prácticas inadecuadas de manejo forestal en el pasado cuando Fábricas de Papel Tuxtepec (Fapatux) utilizaba el Método Mexicano de Ordenación de Bosques Irregurales (MMOBI), cuya corta selectiva propició un cambio en la composición de los bosques al reducir la dominancia de pinos y su reemplazo por encinos de acuerdo con Uzachi (2003). No obstante, un análisis histórico de los últimos 50 años realizado por Gorgonio (2015), señala un incremento en las poblaciones de encino en la Sierra Norte a partir de la década de 1980, período en el que termina la concesión a Fapatux e inicia el manejo forestal comunitario. Esta evidencia, en conjunto con la encontrada por Castellanos-Bolaños et al. (2008) y Ramírez-Santiago (2015) exponen el efecto del manejo forestal en la estructura del dosel, sin embargo, se requiere una mayor investigación para concluir qué tipo de manejo forestal (Fapatux o Comunitario) causó la reducción de 
pinos no comerciales. No obstante, en el futuro se puede causar una reducción de especies de pinos no comerciales, debido a que actualmente los programas de manejo del municipio y la región utilizan como tratamiento silvícola la matarrasa en franja en donde se extraen todas las especies presentes y posteriormente se realizan plantaciones con especies comerciales como P. patula o P. pseudostrobus. Con dichas coníferas se realizan también los programas de reforestación de áreas plagadas o afectadas por incendios, lo que incrementa la abundancia y distribución de estas especies.

Adicionalmente, los procesos históricos de cambio de uso de suelo en el municipio (zonas agrícolas y asentamientos humanos) (Marcial-Juárez, 2011) son la causa de ausencia de pinos en $42 \%$ de las parcelas estudiadas, y puede ser otra razón de la baja abundancia de pinos en zonas agrícolas abandonadas, donde se reforestó con especies comerciales. Esta práctica, en conjunto con un manejo forestal inadecuado constituyen factores que restan superficie a los bosques (Challenger, 1998; SáenzRomero, Snively y Lindig-Cisneros, 2003; Richardson et al., 2007), que en sinergia con factores ambientales modifican la composición y estructura forestal (Perry, 1991; Sánchez-González, 2008).

Por otra parte, la compleja topografía del territorio nacional, unida a las grandes diferencias a nivel regional en latitud y altitud han dado forma a un mosaico climático y ecológico favorable para la gran diversidad de pinos y distribución de las especies que existe en México (Perry, 1991; Bermejo y Pontones, 1999). Capulálpam de Méndez, cuenta con una accidentada topografía y altitudes que varían desde los $1600 \mathrm{~m}$ hasta los $3000 \mathrm{~m}$ snm, intervalo altitudinal en el que se sitúa la distribución de los pinos en el país (Bermejo y Pontones, 1999; del Castillo et al., 2004; Sánchez-González, 2008).

Diversos estudios demuestran que tanto las variables geográficas (altitud y latitud) como climáticas (precipitación y temperatura) influyen en la abundancia y distribución de las especies forestales (e.g. Álvarez-Moctezuma, Ochoa-Gaona, de Jong y Soto-Pinto, 1999; Poulos y Camp, 2005; Naranjo-Luna, 2014). Los valores de especi- ficidad del hábitat y amplitud ecológica de las especies de pinos indican de manera general un efecto de las variables ambientales en la abundancia y distribución de las especies de pinos en Capulálpam de Méndez. En algunas especies como el caso de P. patula, los valores pueden estar sobreestimados debido a que su distribución ha sido incrementada debido a las plantaciones en áreas de bajo aprovechamiento, y su marginalidad y amplitud ecológica puede ser más reducida, similar a las demás especies.

La afinidad ecológica e intervalo de tolerancia de los pinos a variables ambientales puede ser relevante al momento de seleccionar a las especies para plantaciones o restauración con especies forestales para establecerlas en su nicho adecuado, particularmente si a futuro se elige un sistema de manejo forestal cercano a lo natural, en donde se predomina la diversidad de especies, y no monocultivos forestales (Bannister y Pyttel, 2010). Adicionalmente, es importante saber cuáles especies son sensibles a factores climáticos para tomar decisiones sobre estrategias de mitigación contra el cambio climático. Un estudio realizado por Gómez-Mendoza y Arriaga (2007) concluye que la distribución a nivel nacional $P$. rudis, especie presente en Capulálpam de Méndez, es uno de los pinos más vulnerables en México, del que se pronostica una disminución de su distribución de entre $43 \%$ y hasta $64 \%$, que coincide con la alta marginalidad y baja amplitud obtenida en este estudio.

La reducción de la distribución de especies forestales descrita a nivel nacional por Gómez-Mendoza y Arriaga (2007); revela un escenario menos radical en la disminución en superficie de otras especies $(<36 \%)$, por ejemplo, P. douglasiana, cuya reducción sería de $1 \%$ a $2 \%$ de su área actual. Sin embargo, este escenario nacional debe tomarse de manera precautoria en el municipio de Capulálpam de Méndez, dada la sensibilidad de las especies a factores climáticos y donde variaciones en temperatura y precipitación a una escala pequeña, puede tener efectos más severos que a nivel nacional. En este contexto CruzCárdenas et al. (2016) encontraron, a nivel regional, decrementos drásticos mayores a $60 \%$ en el área de distribución de coníferas sensibles como $P$. douglasiana, $P$. rudis, P. leiophylla, P. teocote y P. devoniana. 
Las especies anteriores se encuentran también presentes en el área de estudio, donde muestran una vulnerabilidad mediana a alta a procesos de cambio ambiental. En Sierra Juárez, región donde se localiza Capulálpam de Méndez se registran cambios en el régimen pluviométrico e incremento en la temperatura promedio $\left(\approx 0.3^{\circ} \mathrm{C}\right)$ en las últimas tres décadas (Clark-Tapia, Suárez-Mota, MatíasNolasco y Velasco-Hipólito, 2016). Este cambio ambiental asociado con la alta marginalidad de coníferas incrementa la vulnerabilidad de las especies forestales sensibles, un aspecto que debe considerarse en los planes de manejo forestal del municipio y de la región, de donde se extraen todas las especies del área de aprovechamiento, pero solo se promueve la reforestación con especies comerciales. Lo anterior puede incidir de manera directa en la reducción de las poblaciones y su área de distribución, por lo que se sugiere retener especies de bajo valor comercial, para conservar el nicho de especies asociadas y evitar la pérdida a nivel local de una especie forestal.

\section{CONCLUSIONES}

El municipio de Capulálpam de Méndez presenta una alta riqueza de especies de pinos, dentro las cuales Pinus patula presenta la mayor abundancia y distribución, en contraste con $P$. chiapensis y P. leiophylla que es menor. El manejo forestal, ha generado cambios en la estructura de la vegetación, reflejándose en la dinámica poblacional de las especies forestales en el municipio, lo cual favorece a las especies de alto valor comercial como $P$. patula y $P$. pseudostrobus. De manera general, las especies presentaron requerimientos ambientales específicos y alta sensibilidad a procesos de cambio ambiental, por lo que se recomienda la retención de especies de bajo valor comercial en áreas de aprovechamiento forestal, y la utilización de un sistema silvícola cercano a lo natural, donde se promueva la reforestación mixta de especies.

\section{RECONOCIMIENTOS}

De manera especial se agradece a las Autoridades de Bienes Comunales de Capulálpam y Unión de Comunidades Productoras Forestales Zapotecas-Chinantecas de la Sie- rra Juárez por todo el apoyo proporcionado en la realización de la investigación. Este trabajo fue desarrollado gracias al financiamiento otorgado a través del fondo sectorial de investigación SEP-Conacyt, proyecto 180790, clave institucional 2-EA-0807.

\section{REFERENCIAS}

AddinSoft. (2014). XLSTAT (versión 2014.3.02). BroNY, USA: Addinsoft, Inc.

Álvarez-Moctezuma, J. G., Ochoa-Gaona, S., De Jong, B. H. J. y Soto-Pinto, M. L. (1999). Hábitat y distribución de cinco especies de Quercus (Fagaceae) en la Meseta Central de Chiapas. Biología Tropical, 47, 351-358.

Bannister, J. R. y Pyttel, P. (2010). Silvicultura cercana a lo natural (SCN): "Una nueva visión para el sector forestal chileno?". Bosque Nativo, 46, 26-34.

Bermejo, V. B. y Pontones, B. J. (1999). Los pinos mexicanos y su utilización como especies introducidas de alto potencial en varios países del mundo. ( $2^{\mathrm{do}}$ simposio sobre avances en la producción de semillas forestales en América Latina, memorias 1999). Santo Domingo, Republica Dominicana.

Bray, D. B. y Merino, P. L. (2004). La experiencia de las comunidades forestales en México. Recuperado de: www2.fiu. edu/ brayd/Bray\%20and\%20Merino-La\%20Experiencia.pdf

Castellanos Bolaños, J. F., Treviño Garza, E. J., Aguirre Calderón, O. A., Jiménez Pérez, J., Musalem Santiago, M. y López Aguillón, R. (2008). Estructura de bosque de pino pátula bajo manejo forestal en Ixtlán de Juárez, Oaxaca, México. Madera y Bosques, 14 (2), 51-63.

Clark-Tapia, R., Suárez-Mota, M. E., Matías-Nolasco, M. y Velasco-Hipólito, F. (2016). Clima: pasado, presente y futuro. En R. Clark-Tapia, M. F. Ramos-Morales, C. Alfonso-Corrado, M. M. Mendoza-Díaz y M. FuenteCarrasco (Eds.), Recursos hídricos de la Sierra Norte de Oaxaca: caracterización, diagnóstico y gestión (38-47). México: Universidad de la Sierra Juárez.

Challenger, A. (1998). Utilización y conservación de los ecosistemas terrestres de México; pasado, presente y futuro. México: CONABIO, Instituto de Biología UNAM, Agrupación Sierra Madre, S.C. 
Chapman, S.B. (1976). Methods in plant ecology. Oxford: Blackwell Science Ltd.

Comisión Nacional Forestal (Conafor). (2007a). Ficha técnica para Pinus oaxacana. Recuperado de: http://www. conafor.gob.mx: $8080 /$ documentos/ docs/13/973Pinus\%20oaxacana.pdf.

Comisión Nacional Forestal (Conafor). (2007b). Ficha técnica para Pinus rudis. Recuperado de: http://www.conafor.gob. mx:8080/documentos/docs/13/982Pinus\%20rudis.pdf.

Cruz-Cárdenas, G., López-Mata, L., Silva, J. T., Bernal-Santana, N., Estrada-Godoy, F. y López-Sandoval, J. A. (2016). Potential distribution model of Pinaceae species under climate change scenarios in Michoacán. Revista Chapingo Serie Ciencias Forestales y del Ambiente, 22 (2), 135-148.

Del Castillo, R. F., Pérez de la Rosa, J. A., Vargas-Amado, G. y Rivera-García, R. (2004). Coníferas. En A. J. García Mendoza, M. J. Ordóñez y M. Briones Salas (Eds.), Biodiversidad de Oaxaca (141-158). México.

Díaz, V., Sosa-Ramírez, J. y Pérez-Salicrup, D. R. (2012). Distribución y abundancia de las especies arbóreas y arbustivas en la Sierra Fría, Aguascalientes, México. Polibotánica, 34, 99-126.

Dolédec, S., Chessel, D. y Gimaret-Carpentier, C. (2000). Niche separation in community analysis: a new method. Eco$\log y, 81,2914-2927$.

Enríquez-Enríquez, E. D., Koch, S. D. y González-Elizondo, M. (2003). Flora y vegetación de la Sierra de órganos, municipio de Sombrerete, Zacatecas, México. Acta Botánica Mexicana, 64, 45-89.

Environmental Scientific Research Institute (ESRI). (2000). ArcView (3.x). Redlands, California: ESRI.

Farjon, A. K. y Styles, B. T. (1997). Pinus (Pinaceae). Flora Neotropica Monograph, 75, 1-291.

Gómez-Mendoza, L. y Arriaga, L. (2007). Modeling the effect of climate change on the distribution of oak and pine species of Mexico. Conservation Biology, 21, 1545-1555.

Gorgonio, R. M. (2015). Ecología y genética de Quercus crassifolia (Fagaceae) en sitios de manejo forestal y de uso local en Sierra Juárez. Tesis de maestría no publicada. Universidad de la Sierra Juárez. Ixtlán de Juárez, Oaxaca, México.
Hammer, Q., Harper, D. A. T. y Ryan, P. D. (2001). PAST: Paleontological statistics software package for education and data analysis. Paleontología Electrónica, 4 (1), 1-9.

Hernández-Díaz, J. C., Corral-Rivas, J. J., Quiñones-Chávez, A., Bacon-Sobbe, J. R. y Vargas-Larreta, B. (2008). Evaluación del manejo forestal regular e irregular en bosques de la Sierra Madre Occidental. Madera y Bosques, 14 (3), 25-41.

Hijmans, R. J., Cameron, S. E., Parra, J. L., Jones, P. G. y Jarvis, A. (2005). Very high resolution interpolated climate surfaces for global land areas. International Journal of Climatology, 25, 1965-1978.

Hurlbert, A. H. y White, E. P. (2007). Ecological correlates of geographical range occupancy in North American birds. Global Ecology and Biogeography, 16, 764-773.

Instituto Nacional de Estadística, Geografía e Informática (Inegi). (2001). Conjunto de Datos Vectoriales. Santa Catarina Ixtepeji E14D38. Escala 1: 50,000, Oaxaca, México.

Jardel, E. J. (2012). El manejo forestal en México: estado actual y perspectivas. En F. Chapela (Ed.), Estado de los Bosques en México (69-115). México: Consejo Civil Mexicano para la Silvicultura Sostenible.

Kimmins, J. P. (2003). Forest ecology: a foundation for sustainable forest management and environmental ethics in forestry. Minnesota, USA: Benjamin Cummings, Pearson.

Lavergne, S., Molina, W. J. y Debussche, M. (2005). Environmental and human factors influencing rare plant local occurrence, extinction and persistence: a 115-year study in the Mediterranean region. Journal of Biogeography, 32, 799-811.

Marcial-Juárez, R. (2011). Cambio de uso de suelo en Capulálpam de Méndez. Tesis de licenciatura no publicada. Universidad de la Sierra Juárez. Ixtlán de Juárez, Oaxaca, México.

Márquez-Linares, M. A., González-Elizondo, S. y ÁlvarezZagoya, R. (1999). Componentes de la diversidad arbórea en bosques de pino encino de Durango, México. Madera $y$ Bosques, 5 (2), 67-78.

Meier, E.S., Lischke, H. Schmatz, D. R. y Zimmermann, N. E. (2011). Climate, competition and connectivity affect 
future migration and ranges of European trees. Global Ecology and Biogeography, 21, 164-178.

Murcia, C. (1995). Edge effects in fragmented forests: implications for conservation. Tree, 10, 58-62.

Naranjo-Luna, F. J. (2014). Ecología y genética de Oreomunnea mexicana (Standl.) J.F. Leroy (Junglandaceae), especie relicto del bosque de niebla de la Sierra Juárez, Oaxaca. Tesis de maestría no publicada. Universidad de la Sierra Juárez. Ixtlán de Juárez, Oaxaca, México.

Plan de Desarrollo Municipal (PDM). (2009). Plan de Desarrollo Municipal Capulálpam de Méndez. Oaxaca, México.

Perry, J. P. (1991). The pines of México and Central America. Portland, Oregon, EUA: Timber Press.

Perry, J. P., Graham, A. y Richardson M. D. (1998). The history of pines in México and Central America. En M.D. Richardson (Ed.). Ecology and Biogeography of Pinus (137-149). Cambridge, UK: Cambridge University Press.

Poulos, H. M. y Camp, A. E. (2005). Vegetation - Environment relations of the Chisos Mountains, Big Bend National Park, Texas. Fort Collins: USDA Forest Service Proceedings RMRS.

Ramírez Santiago, R. (2015). Estructura y dinámica de un bosque de pino-encino bajo manejo forestal en la Sierra Norte de Oaxaca. Disertación doctoral. Colegio de Postgraduados, Campus Montecillo. Texcoco, Estado de México.

Richardson, D. M., Rundel, P. W., Jackson, S. T., Teskey, R. O., Aronson, J., Bytnerowicz, A., Wingfield, M. J. y Proches, S. (2007). Human impacts in pine forests: past, present and future. Annual Review of Ecology, Evolution, and Systematics, 38, 275-297.

Sáenz-Romero, C., Snively, A. E. y Lindig-Cisneros, R. (2003). Conservation and restoration of pine forest genetic resources in Mexico. Silvae Genetica, 52, 5-6.
Sánchez-González, A. (2008). Una visión actual de la diversidad y distribución de los pinos de México. Madera y Bosques, 14 (1), 107-120.

Solís-Moreno, R., Aguirre-Calderón, O. A., Treviño-Garza, E. J., Jiménez-Pérez, J., Jurado-Ybarra, E. y Corral-Rivas, J. (2006). Efecto de dos tratamientos silvícolas en la estructura de ecosistemas forestales en Durango, México. Madera y Bosques, 12 (2), 49-64.

Thiolouse, J., Chessel, D., Dóledec, S. y Olivier, J. M. (1997). ADE-4: a multivariate analysis and graphical display software. Statistics and Computing, 7, 75-83.

Thuiller, W., Gassó, N., Pino, J. y Vilá, M. (2012). Ecological niche and species traits: key drivers of regional plant invader assemblages. Biological Invasions, 14, 1963-1980.

Tsiftsis, S., Tsiripidisa, I., Karagiannakidoua, V. y Alifragisb, D. (2008). Niche analysis and conservation of the orchids of East Macedonia (NE Greece). Acta Oecologica, 33, 27-35.

Unión de comunidades productoras forestales Zapotecas y Chinantecas (Uzachi). (2003). Programa de manejo forestal persistente para el aprovechamiento maderable de la comunidad de Capulálpam de Méndez, Ixtlán, Oaxaca.

Van Zonneveld, M., Koskela, J., Vinceti, B. y Jarvis, A. (2009). Repercusiones del cambio climático en la distribución de los pinos tropicales en Asia sudoriental. Unasylva, 60, 24-28.

Manuscrito recibido el 15 de julio de 2015.

Aceptado el 13 de septiembre de2016.

Este documento se debe citar como:

Ríos-Altamirano, A., Alfonso-Corrado, C., Aguirre-Hidalgo, V., Ángeles-Pérez, G., Mendoza-Díaz, M. M., Rodríguez-Rivera, V., RoldánFelix E. y Clark-Tapia, R. (2016). Abundancia y distribución del género Pinus en Capulálpam de Méndez, Sierra Juárez, Oaxaca. Madera y Bosques, 22 (3), 61-74. 